\title{
Nano Particles @ Calix Arenas Via Aqueous Solution
}

\author{
SAHAR DEHGHANI \\ Department of Chemistry, Science and Research Branch, Islamic Azad University, Tehran, Iran. \\ ${ }^{*}$ Corresponding author E-mail: sahardehghani27@yahoo.com \\ http://dx.doi.org/10.13005/ojc/320224
}

(Received: February 03, 2016; Accepted: March 07, 2016)

\begin{abstract}
The electronic structure and magnetic properties of $\mathrm{Al}_{2} \mathrm{O}_{3}, \mathrm{GaN}$ and $\mathrm{Fe}_{3} \mathrm{O}_{4} @$ Calix (8) $\mathrm{COOH}$ have been studied using ONIOM and DFT methods. The studies focus on how to improve the adsorption of some nano particles solution aqueous for achieving good magnetic and functionalized potential performances. The results revealed that the Fe3O4@ Calix (8) $\mathrm{COOH}$ and some of its derivations exhibited better thermodynamic stability. Furthermore, the particle size and magnetic property of the GaN@ Calix (8) $\mathrm{COOH}$ nanoparticles can be controlled by the aqueous. The electrical properties such as NMR Shielding, electron densities, energy densities, potential energy densities, ELF, LOL, ellipticity of electron density, eta index and ECP for nanoparticles@ Calix (8)COOH have been calculated.
\end{abstract}

Keyword: superparamagnetic nano particle, QM/MM simulation, Calix (n).

\section{INTRODUCTION}

What makes GaN popular is not only it is more robust but it can emit in the short wavelength part of the visible spectrum. For example, there are now GaN-based high-efficiency blue and green light-emitting diodes ${ }^{1}$ And just arenes when researchers thought things could not get better for $\mathrm{GaN}$, a whole new field of research has opened up in the form of $\mathrm{GaN}$ nanotubes ${ }^{2}$. These nanotubes were synthesized by an" epitaxial casting" strategy by the research group of Peidong Yang at Lawrence Berkeley National Lab oratory. ZnO nanowires, grown on sapphire wafers, were used as templates for epitaxial overgrowth of thin GaN layers in a chemical vapor Deposition (CVD) system. The result of electron diffraction measurements showed that the nanotubes obtained in this way are single-crystal, and in this respect they are fundamentally different from theoretically simulated GaN nanotubes ${ }^{3}$ with the conventional tubular forms of carb on atoms ${ }^{4}$.

On the other hand, most of the applications require magnetic particles to disperse in a nonmagnetic matrix. The matrixes play an important role in determining physical properties of the composite 
nanoparticle in addition to providing a means of particle dispersion ${ }^{5}$.

$\mathrm{Fe}_{3} \mathrm{O}_{4}$ is an electrical conductor with conductivity significantly higher than $\mathrm{Fe}_{2} \mathrm{O}_{3}$, and this is ascribed to electron exchange between the Fe" and $\mathrm{Fe}^{\mathrm{III}}$ centers $^{8-11}$.

$\mathrm{Fe}_{3} \mathrm{O}_{4}$ is ferromagnetic with a curie temperature of $858 \mathrm{~K}$ and The ferromagnetism of $\mathrm{Fe}_{3} \mathrm{O}_{4}$ arises because the electron spins of the $\mathrm{Fe}^{\mathrm{II}}$

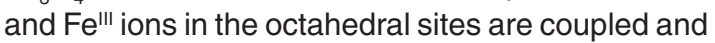
the spins of the Fe ${ }^{\text {III }}$ ions in the tetrahedral sites are coupled but anti-parallel to the former ${ }^{4-7}$.

Nano-Magnetites have shown great potential applications in the field of in vitro and in vivo biomedicine, including cellular therapy in cell labeling, separation and purification, target-drug delivery, and hyperthermia treatment of cancers.

Magnetic nanoparticles have attracted much interest not only in the field of magnetic recording but also in the areas of medical field of magnetic sensing. Especially, nanoparticles of iron oxide are reported to be applicable as a material for use in drug delivery systems, magnetic resonance imaging, and cancer therapy ${ }^{5-8}$.

$\mathrm{Fe}_{3} \mathrm{O}_{4}$ is used as a catalyst in the Haber process and in the water gas shift reaction ${ }^{12,13}$.

The latter uses an HTS (high temperature shift catalyst) of iron oxide stabilized by chromium oxide $^{11,12}$. This iron-chrome catalyst is reduced at reactor start up to generate $\mathrm{Fe}_{3} \mathrm{O}_{4}$ from á- $\mathrm{Fe}_{2} \mathrm{O}_{3}$ and $\mathrm{Cr}_{2} \mathrm{O}_{3}$ to $\mathrm{CrO}_{3}{ }^{13}$.

In this work the $\mathrm{Al}_{2} \mathrm{O}_{3}, \mathrm{GaN}$ and $\mathrm{Fe} 3 \mathrm{O} 4 @$ Calix (8) $\mathrm{COOH}$ have been investigated and the catalysis's properties of these nanoparticles have been studied

The data have been compared with the those nanoparticles @ CWCNNTs and BNNTs Carbon nanotube (CNT) is a representative nanomaterial. CNT is a cylindrically shaped carbon material with a nano-metric-level diameter ${ }^{11-38}$. Its structure, which is in the form of a hexagonal mesh, resembles a graphite sheet and it carries a carbon atom located on the vertex of each mesh. The sheet has rolled and its two edges have connected seamlessly ${ }^{39-45}$.

Although it is a commonplace material using in pencil leads, its unique structure causes it to present characteristics that had not found with any other materials. CNT can be classified into single-wall CNT, double-wall CNT and multi-wall CNT according to the number of layers of the rolled graphite ${ }^{46,70}$.

The type attracting most attention is the single-wall CNT, which has a diameter deserving the name of "nanotube" of 0.4 to 2 nanometers. The length is usually in the order of microns, but singlewall CNT with a length in the order of centimeters has recently released.

CNT can be classified into single-wall CNT, double-wall CNT and multi-wall CNT according to the number of layers of the rolled graphite. The type attracting most attention is the single-wall CNT, which has a diameter deserving the name of "nanotube" of 0.4 to 2 nanometers ${ }^{51-69}$.

The length is usually in the order of microns, but single-wall CNT with a length about centimeters have recently released. The extremities of the CNT have usually closed with lids of the graphite sheet $^{70-85}$.

The lids consist of hexagonal crystalline structures (six-membered ring structures) and a total of six pentagonal structures (five-membered ring structures) placed here and there in the hexagonal structure $^{86-99}$. The first report by lijima was on the multiwall form, coaxial carbon cylinders with a few tens of nanometers in outer diameter. Two years later single walled nanotubes were reported ${ }^{80-100}$. SWBNNTs have considered as the leading candidate for nano-device applications because of their onedimensional electronic bond structure, molecular size, and biocompatibility, controllable property of conducting electrical current and reversible response to biological reagents hence SWBNNTs make possible bonding to polymers and biological systems such as DNA and carbohydrates ${ }^{100-133}$. 


\section{Computational details}

Part of the systems including $\mathrm{GaN}, \mathrm{Al}_{2} \mathrm{O}_{3}$ and $\mathrm{Fe}_{3} \mathrm{O}_{4} @$ Calix (8)COOH, and @ SWBNNNTs and SWCNTs have been modeled with ONIOM method and the calculations are carried out with the Molecular Mechanics methods. In this investigation, differences in force field are illustrated by comparing the calculated energy with CHARMM, AMBER and OPLS force fields. Furthermore, a HyperChem professional release 7.01 programs is used for the additional calculations.

For non-covalent interactions between nano particles and Calix (8)COOH , the B3LYP method is unable to describe van der Waals by medium-range interactions. Therefore, the ONIOM methods including 3 levels of 1-high calculation $(H)$, 2-medium calculation (M), and 3-low calculation (L) have been performed in our study for calculating the non-bonded interactions between nanoparticles and Calix (8) $\mathrm{COOH}$.

The ab-initio and DFT methods are used for the model system of the ONIOM layers and the semi empirical methods of pm6 (including pseudo=lanl2) and Pm3MM are used for the medium and low layers, respectively.

B3LYP and the most other popular and widely used functional are insufficient to illustrate the exchange and correlation energy for distant nonbonded medium-range systems correctly. Moreover, some recent studies have shown that inaccuracy for the medium-range exchange energies leads to large systematic errors in the prediction of molecular properties.

Table1: All Electron Densities Of Non-Bonded Interactions For $\mathrm{Al}_{2} \mathrm{O}_{3} @$ Calix [8]COOH

\begin{tabular}{lcccc}
\hline $\begin{array}{l}\text { Atom } \\
\text { (number) }\end{array}$ & $\begin{array}{c}\text { Density of all } \\
\text { electron(10 }\end{array}$ & $\begin{array}{c}\text { Density of } \\
\text { alpha }\left(\mathbf{1 0}^{-3}\right)\end{array}$ & $\begin{array}{c}\text { Density of } \\
\text { Beta (10-3) }\end{array}$ & $\begin{array}{c}\text { Spin } \\
\text { Density }\end{array}$ \\
\hline $\mathrm{Al}(1)$ & 0.18 & 0.09 & 0.09 & 0.0 \\
$\mathrm{Al}(2)$ & 0.18 & 0.09 & 0.09 & 0.0 \\
\hline
\end{tabular}

Table 2: All Electron Energies of non-bonded interactions for $\mathrm{Fe}_{3} \mathrm{O}_{4} @$ Calix [8] COOH

\begin{tabular}{|c|c|c|c|c|}
\hline $\begin{array}{l}\text { Atom } \\
\text { (number) }\end{array}$ & \multicolumn{2}{|c|}{$\begin{array}{l}\text { Lagrangian kinetic } \\
{[\mathrm{G}(\mathrm{r})] \text { energy }\left(10^{-3}\right)}\end{array}$} & \multicolumn{2}{|c|}{$\begin{array}{l}\text { Hamiltonian kinetic } \\
{[\mathrm{K}(\mathrm{r})] \text { energy }\left(10^{-2}\right)}\end{array}$} \\
\hline $\mathrm{Fe}(1)$ & \multicolumn{2}{|c|}{0.34} & \multicolumn{2}{|c|}{0.41} \\
\hline $\mathrm{Fe}(2)$ & \multicolumn{2}{|c|}{0.36} & \multicolumn{2}{|c|}{0.52} \\
\hline $\mathrm{Fe}(3)$ & \multicolumn{2}{|c|}{0.32} & \multicolumn{2}{|c|}{0.41} \\
\hline \multicolumn{5}{|c|}{$\begin{array}{l}\text { Table 3: Laplacian, ELF, LOL and Local information entropy of non- } \\
\text { bonded interactions for } \mathrm{Fe}_{3} \mathrm{O}_{4} @ \text { Calix }[8] \mathrm{COOH}\end{array}$} \\
\hline $\begin{array}{l}\text { Atom } \\
\text { (number) }\end{array}$ & $\begin{array}{l}\text { Laplacian of } \\
\text { electron } \\
\text { density }\left(10^{-1}\right)\end{array}$ & $\begin{array}{r}\text { Ele } \\
\text { localizati } \\
(\text { ELF }\end{array}$ & $\begin{array}{l}\text { unction } \\
\left.0^{-3}\right)\end{array}$ & $\begin{array}{l}\text { Local } \\
\text { information } \\
\text { entropy }\left(10^{-4}\right)\end{array}$ \\
\hline $\mathrm{Fe}(1)$ & -0.12 & & & 0.33 \\
\hline $\mathrm{Fe}(2)$ & -0.41 & & & 0.41 \\
\hline $\mathrm{Fe}(3)$ & -0.38 & & & 0.12 \\
\hline
\end{tabular}


Geometry optimizations and electronic structure calculations have been carried out using the m06 (DFT) functional. This approach is based on an iterative solution of the Kohn-Sham equation of the density functional theory in a plane-wave set with the projector-augmented wave pseudo-potentials. The Perdew-Burke-Ernzerhof (PBE) exchangecorrelation $(X C)$ functional of the generalized gradient approximation (GGA) is also used. The optimizations of the lattice constants and the atomic coordinates are made by the minimization of the total energy.

The charge transfer and electrostatic potential-derived charge were also calculated using the Merz-Kollman-Singh chelp or chelpG the charge calculation methods based on molecular electrostatic potential (MESP) fitting are not wellsuited for treating larger systems whereas some of the innermost atoms are located far away from the points at which the MESP is computed. In such a condition, variations of the innermost atomic charges will not head towards a significant change of the MESP outside of the molecule, meaning that the accurate values for the innermost atomic charges are not well-determined by MESP outside the molecule . The representative atomic charges for molecules should be computed as average values over several molecular conformations.
A detailed overview of the effects of the basis set and the Hamiltonian on the charge distribution can be found in references. The charge density proûles in this study has been extracted from ûrst-principles calculation through an averaging process as described in reference ${ }^{122-124}$. The interaction energy for capacitor was calculated in all items according to the equation as follows:

$\Delta \mathrm{E}_{\mathrm{S}}(\mathrm{eV})=\left\{\mathrm{E}_{\mathrm{c}}-\left(\sum_{(\mathrm{i}=1)}^{\mathrm{n}}(\right.\right.$ Nanoparticles- $(\mathrm{n}, \mathrm{n})$ SWBNNTs $+\sum_{(i=6)}^{14}$ (nanoparticles-(n,n)SWBNNTs)\}

Where the " $\Delta \mathrm{E}_{\mathrm{S}}$ " is the stability energy. The electron density, electron spin density, electron localization function (ELF), total electrostatic potential (ESP), value of orbital wave-function, electrostatic potential from nuclear atomic charges and localized orbital locator (LOL) which has been defined by Becke and Tsirelson, as well as the exchange-correlation density, correlation hole and correlation factor, and the average local ionization energy using the Multifunctional Wavefunction analyzer have also been calculated in this study ${ }^{122-124}$. The contour line map was also drawn using the Multiwfn software ${ }^{122,124}$. The solid lines indicate positive regions, while the dash lines indicate negative regions. The contour line corresponding to VdW surface which is defined by R. F. W Bader

Table 4: Average local ionization energy, RDG and ESP of non-bonded interactions for GaN@ Calix [8]

\begin{tabular}{lccc}
\hline $\begin{array}{l}\text { Atom } \\
\text { (number) }\end{array}$ & $\begin{array}{c}\text { Reduced density } \\
\text { gradient(RDG) }\left(\mathbf{1 0}^{+}\right)\end{array}$ & $\begin{array}{c}\text { Average local } \\
\text { ionization energy }\end{array}$ & $\begin{array}{c}\text { ESP from } \\
\left.\text { electron charge (10 } \mathbf{2}^{\mathbf{2}}\right)\end{array}$ \\
\hline $\mathrm{Ga}(\mathbf{1})$ & 0.30 & 0.31 & -0.21 \\
$\mathrm{~N}(2)$ & 0.34 & 0.33 & -0.51 \\
\hline
\end{tabular}

Table 5: Lambada2, Wave function value, Ellipticity of electron density and Eta index of non-bonded interactions for $\mathrm{Al}_{2} \mathrm{O}_{3} @$ Calix [8] $\mathrm{COOH}$

\begin{tabular}{lcc}
\hline $\begin{array}{l}\text { Atom } \\
\text { (number) }\end{array}$ & $\begin{array}{c}\text { Wave function } \\
\text { value } \mathbf{1 0}^{-4}\end{array}$ & $\begin{array}{c}\text { Ellipticity of } \\
\text { electron density }\end{array}$ \\
\hline $\mathrm{Al}(1)$ & 0.49 & 0.13 \\
$\mathrm{Al}(2)$ & 0.57 & 0.23 \\
$\mathrm{O}(3)$ & 0.23 & 0.28 \\
\hline
\end{tabular}

is plotted in this study. This is specifically useful to analyze distribution of electrostatic potential on VdW surface.

$$
\begin{aligned}
& \text { Theoretical background } \\
& \text { Electron density } \\
& \text { The electron density has been defined as } \\
& \rho(r)=\eta_{i}\left|\varphi_{i}(r)\right|^{2}=\sum_{i} \eta_{i}\left|\sum_{l} C_{l, i} \chi_{i}(r)\right|^{2}
\end{aligned}
$$


Where $\chi$ is basis function, $\eta \mathrm{i}$ is occupation number of orbital (i), $\varphi$ is orbital wave function, and $C$ is coefficient matrix. Atomic unit for electron density can be explicitly written as:

$$
\nabla \rho(r)=\left[\left(\frac{\partial \rho(r)}{\partial(x)}\right)^{2}+\left(\frac{\partial \rho(r)}{\partial(y)}\right)^{2}+\left(\frac{\partial \rho(r)}{\partial(z)}\right)^{2}\right]^{\frac{1}{2}}
$$

Table 6: All Electron Densities of non-bonded interactions for $\mathrm{Al}_{2} \mathrm{O}_{3}(6,6)$ SWBNNTs

\begin{tabular}{lcccc}
\hline $\begin{array}{l}\text { Atom } \\
\text { (number) }\end{array}$ & $\begin{array}{c}\text { Density of } \\
\text { all electron(10-3) }\end{array}$ & $\begin{array}{c}\text { Density of } \\
\text { alpha (10-3) }\end{array}$ & $\begin{array}{c}\text { Density of } \\
\text { Beta (10-3) }\end{array}$ & $\begin{array}{c}\text { Spin } \\
\text { Density }\end{array}$ \\
\hline $\mathrm{AL}(1)$ & 0.23 & 0.25 & 0.16 & 0.0 \\
$\mathrm{Al}(2)$ & 0.35 & 0.12 & 0.15 & 0.0 \\
\hline
\end{tabular}

Table 7: The properties calculated data for Calix[8,8]COOH

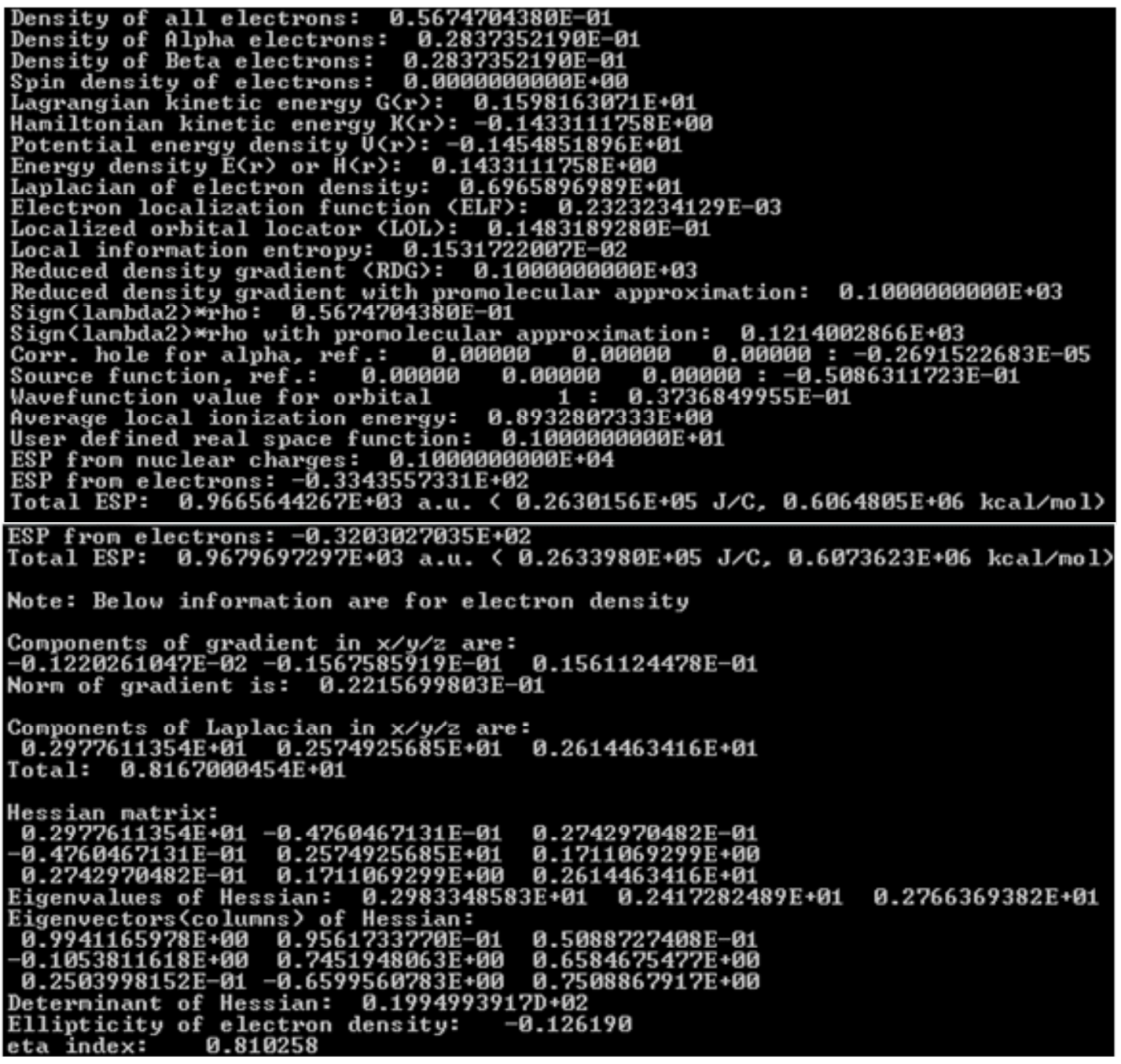



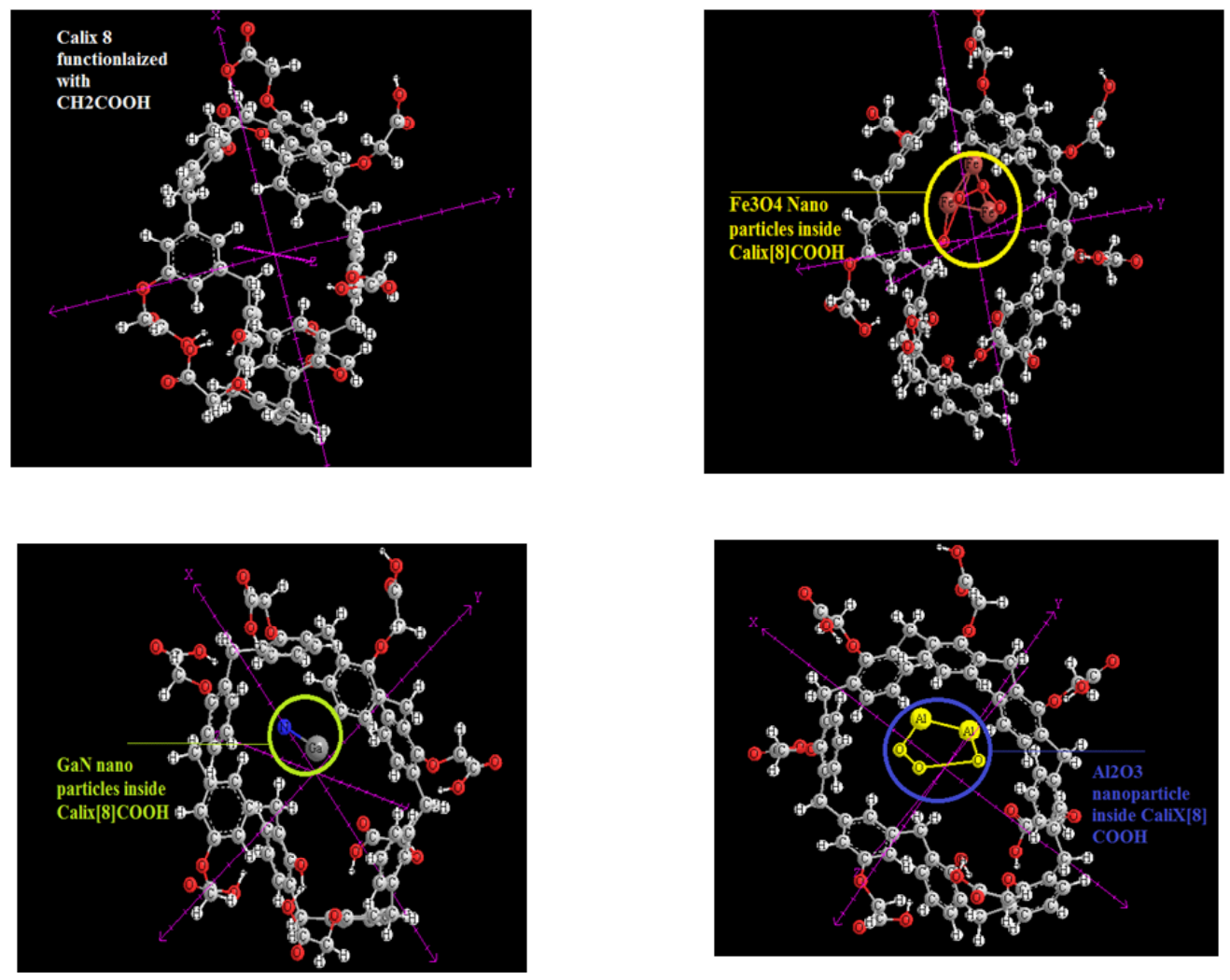

Fig.1: Optimized Calix [8] $\mathrm{COOH}$ including $\mathrm{Fe}_{3}, \mathrm{O}_{4}$, $\mathrm{GaN}$ and Al2O3 nanoparticles with DFT methods

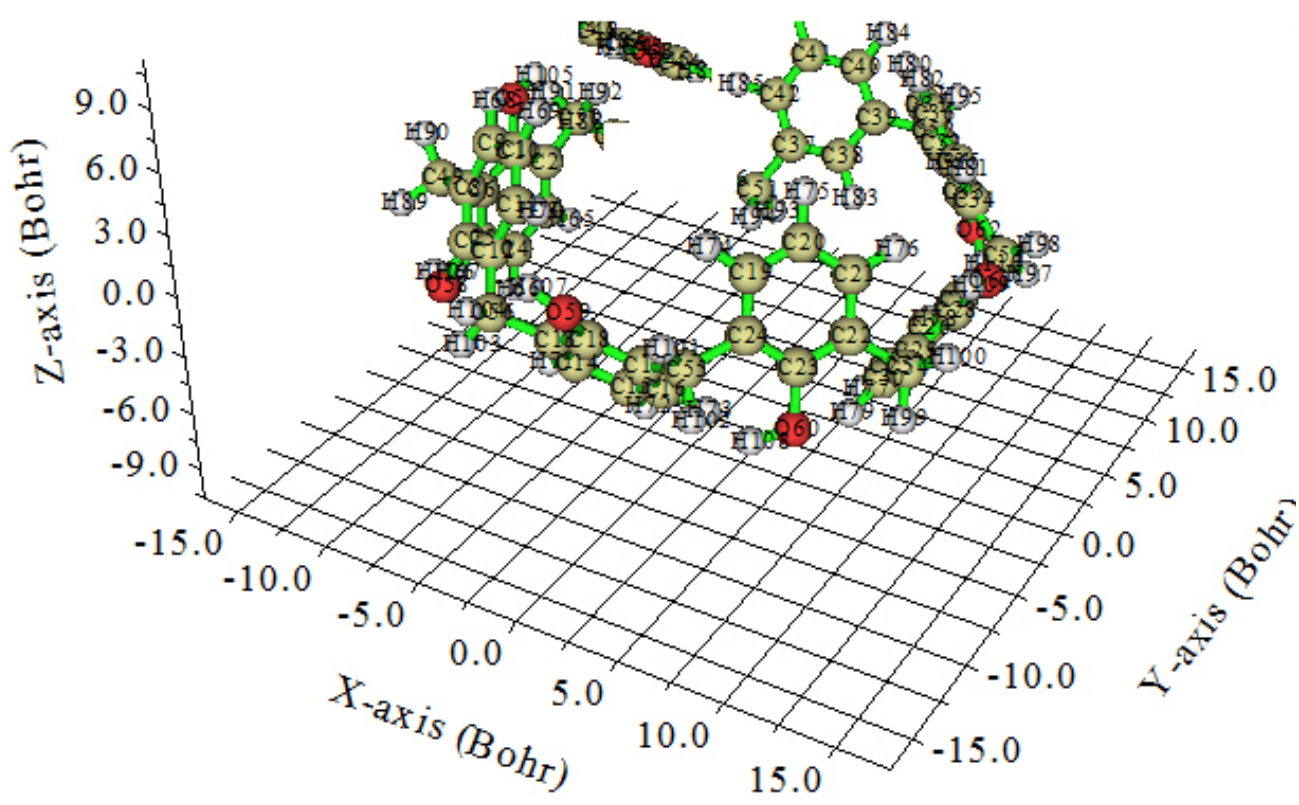

Fig. 2: The Calix $[8,8] \mathrm{COOH}$ situations versus axis 
$\nabla^{2} \rho(r)=\frac{\partial^{2} \rho(r)}{\partial x^{2}}+\frac{\partial^{2} \rho(r)}{\partial y^{2}}+\frac{\partial^{2} \rho(r)}{\partial z^{2}}$

Locally depleted and locally concentrated are the positive and negative value of these functions, respectively. The relationships between $\nabla^{2} \rho$ and valence shell electron pair repulsion or VSEPR model, electron localization, chemical bond type, and chemical reactivity have been investigated by Bader $^{133}$.

The kinetic energy density is not uniquely defined, since the expected value of kinetic energy operator $\left\langle\varphi\left|-\left(\frac{1}{2}\right) \nabla^{2}\right| \varphi\right\rangle$ can be recovered by integrating kinetic energy density from alternative definitions. One of commonly used definition is:

$$
k(r)=-\frac{1}{2} \sum_{i} \eta_{i} \varphi_{i}^{*}(r) \nabla^{2} \varphi_{i}(r)
$$

Lagrangian kinetic energy density, " $G(r)$ " is also known as positive definite kinetic energy Nannit."

$$
\begin{gathered}
G(r)=\frac{1}{2} \sum_{i} \eta_{i} \mid \nabla\left(\left.\varphi_{i}\right|^{2}=\frac{1}{2} \sum_{i} \eta_{i}\left\{\left[\left(\frac{\partial \varphi_{i}(r)}{\partial(x)}\right)^{2}\right.\right.\right. \\
\left.\left.+\left(\frac{\partial \varphi_{i}(r)}{\partial(y)}\right)^{2}+\left(\frac{\partial \varphi_{i}(r)}{\partial(z)}\right)^{2}\right]\right\}
\end{gathered}
$$

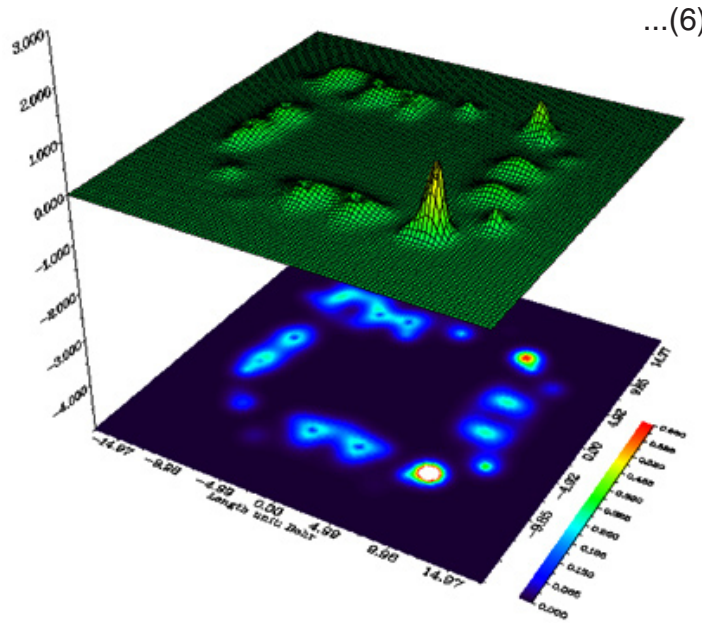

Fig. 3: Shaded map including projection for calix [8] $\mathrm{COOH}$
$K(r)$ and $G(r)$ are directly related by Laplacian of electron density

$$
\frac{1}{4} \nabla^{2} \rho(r)=G(r)-K(r)
$$

Becke and Edgecombe noted that spherically averaged like spin conditional pair probability has direct correlation with the Fermi hole and then suggested electron localization function $(E L F)^{126}$.

$$
E L F(r)=\frac{1}{1+\left[D(r) / D_{0(r)}\right]^{2}}
$$

where

$$
\mathrm{D}(\mathrm{r})=\frac{1}{2} \sum_{i} \eta_{i}\left|\nabla \varphi_{i}\right|^{2}-\frac{1}{8}\left[\frac{\left.|| \nabla \rho_{\alpha}\right|^{2}}{\rho_{\alpha}(r)}+\frac{\left.|| \nabla \rho_{\beta}\right|^{2}}{\beta(r)}\right]
$$

and

$$
D_{0(r)}=\frac{3}{10}\left(6 \pi^{2}\right)^{2}\left[\rho_{\alpha}(r)^{3}+\rho_{\beta}(r)^{3}\right]
$$

for close-shell system, since

$$
\rho_{\alpha}(r)=\rho_{\beta}(r)=\frac{1}{2} \rho, D \text { and } D 0 \text { terms can }
$$
be simplified as

$$
\mathrm{D}(\mathrm{r})=\frac{1}{2} \sum_{i} \eta_{i}\left|\nabla \varphi_{i}\right|^{2}-\frac{1}{8}\left[\frac{|\nabla \rho|^{2}}{\rho(r)}\right]
$$

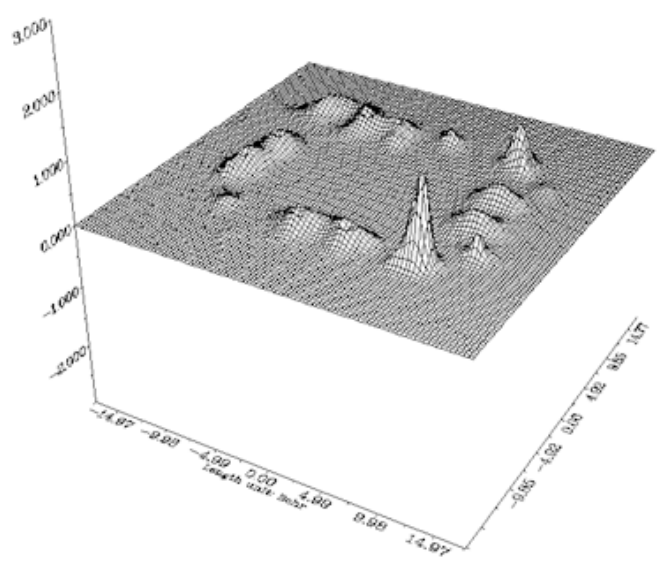

Fig. 4: Relief map for Calix [8]COOH 


$$
D_{0(r)}=\frac{3}{10}\left(3 \pi^{2}\right)^{2} \rho(r)^{3}
$$

Savin et al. have investigated the ELF in the view point of kinetic energy, ${ }^{134}$ which makes ELF also meaningful for Kohn-Sham DFT wave-function or even post-HF wave-function. They indicated that $D(r)$ reveals the excess kinetic energy density caused by Pauli repulsion, while $D 0(r)$ can be considered as Thomas-Fermi kinetic energy density ${ }^{135}$.

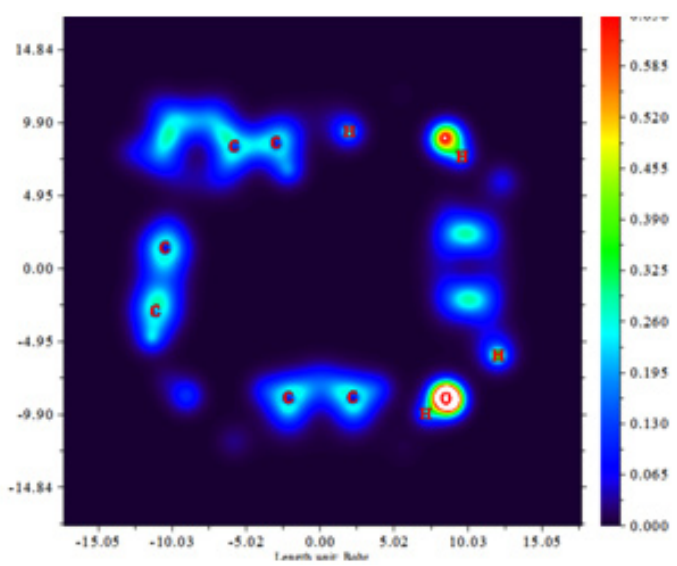

Localized orbital locator (LOL) is another function for locating high localization regions likewise ELF, defined by Schmider and Becke in the paper $^{136}$.

$$
\operatorname{LOL}(r)=\frac{\tau(r)}{1+\tau(r)}
$$

where

$$
(r)=\frac{D_{0}(r)}{\frac{1}{2} \Sigma_{i} \eta_{i}\left|\nabla \varphi_{i}\right|^{2}}
$$

Fig. 5: Color and contour map for the Calix[8]COOH

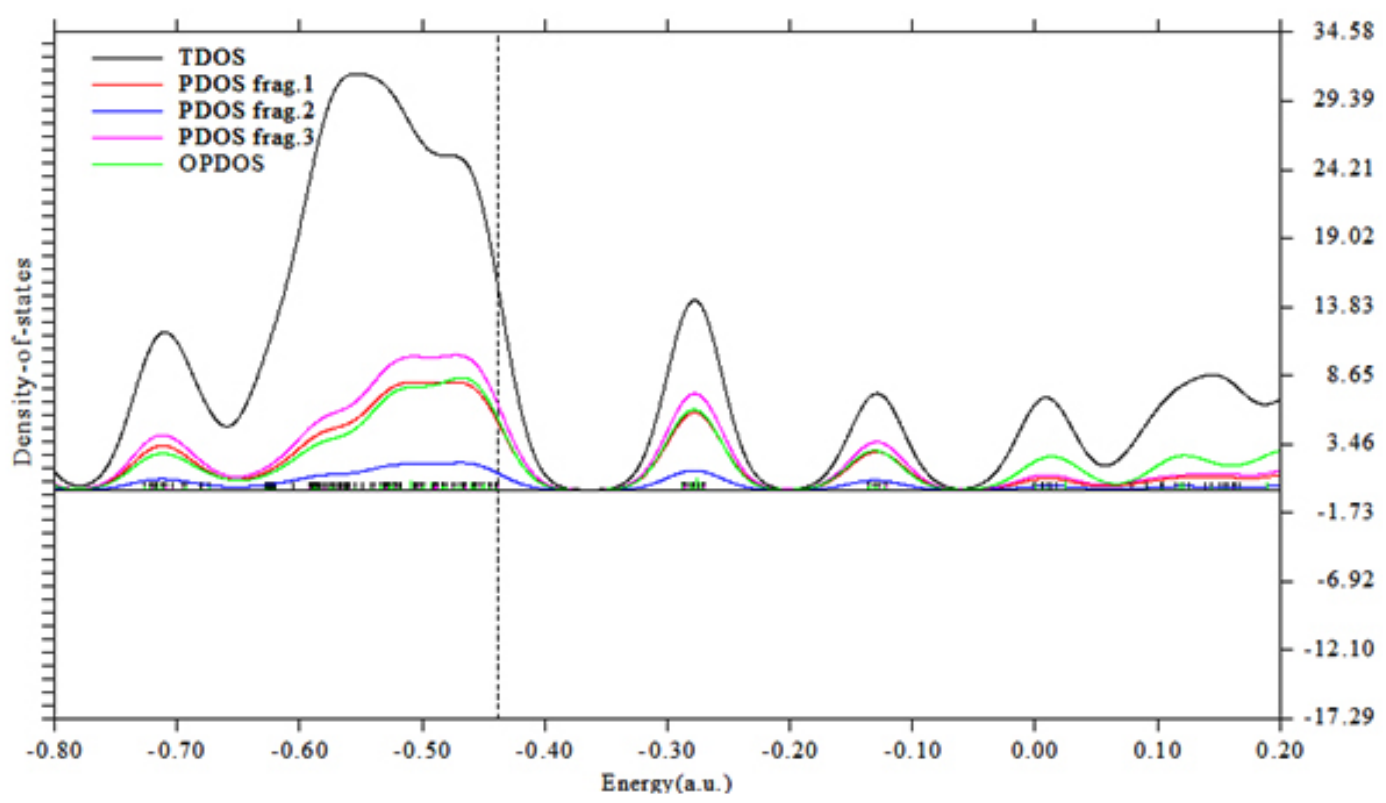

Fig. 6: Density of states for $\mathrm{Fe}_{3} \mathrm{O}_{4} @$ calix [8]COOH including TDOS, PDOS, and OPDOS 
$D_{0}(r)$ for spin-polarized system and closeshell system are defined in the same way as in $\mathrm{ELF}^{137}$

\section{RESULT AND DISCUSSION}

This study mainly focuses on the magnetic properties of nanoparticles in a non-bonded system with Calix (8) $\mathrm{COOH}$ and $(n, n)$ SWCNTs core-shells. The non-bonded interaction is shown in figs 1-7. As it is indicated in tables 1-7, the electrical properties can be obtained from changes in the non-bonded interactions. Electron densities, energy densities, Potential energy densities, ELF, LOL, and Ellipticity of electron density, eta index and ECP for nanoparticles @ Calix (8)COOH and nanoparticles @ SWCNTs were calculated of each simulation (Tables 1-7).

The largest electron localization is located on $\mathrm{Al} 2 \mathrm{O} 3$ where the electron motion is more likely to be confined within that region. If electrons are completely localized in the GaN, they can be distinguished from the ones outside. As shown in tables 1-8 the large ELF is close to the Al2O3 atoms. The regions with large electron localization need to have large magnitudes of Fermi-hole integration which would lead the nanoparticles towards superparamagnetic. The fermi hole is a sixdimension function and as a result, it is difficult to be studied visually. Based on equations 12, 13 and 14,
Becke and Edgecombe noted that the Fermi hole is a spherical average of the spin which is in good agreement with our results in tables and Figs.

ELF indicates that it is actually a relative localization and must be accounted within the range of $[0,1]$. A large ELF value corresponds to largely localized electrons which indicate that a covalent bond, a lone pair or inner shells of the atom is involved. According to equation 16, LOL can be interpreted similar to ELF in terms of kinetic energy, though; LOL can also be interpreted in terms of localized orbitals. Small (large) LOL value usually appears in boundary (inner) region of localized orbitals due to the large (small) gradient of orbital wave-function in this area. The value range of $\mathrm{LOL}$ is identical to ELF, namely $[0,1]$.

The total electrostatic potential (ESP) measures the electrostatic interaction between a unit point charges placed at $r$ and the system of interest. A positive (negative) value implies that current position is dominated by nuclear (electronic) charges. Molecular electrostatic potential (ESP) has been widely used for prediction of nucleophilic and electrophilic sites for a long time.

It is also valuable in studying hydrogen bonds, halogen bonds, molecular recognitions and the intermolecular interaction of aromatics ${ }^{138-142}$

\section{REFERENCES}

1. Nakamura,S.; Mukai,T.; Senoh, M.; Appl. Phys. Lett. 1994, 64, 1687.

2. Goldberger, J.; He, R.: Zhang,Y.; Lee, S.;Yan, H.; J. Choi, H.; Yang, P, Nature (London) ,2003 ,422, 599.

3. S.M. Lee, Y.H. Lee, Y.G. Hwang, J. Elsner, D. Porezag, T. Frauenheim, Phys. Rev.1999, B 60, 7788

4. lijima, S.; Nature (London)1991, 354, 56 (1991).

5. BERRY, C.C.; CURTIS, A. S. G. J Phys D: Appl Phys, 2003, 36: R198"R206.

6. RUUGE, E.K.; RUSETSKI, A. N. J Magn Magn Mater, 1993, 122: 335"339.

7. POPE, N.M.; ALSOP, R. C.; CHANG, Y. A.; SMITH, A. K. J Biomed Mater Res, 1994, 2: 449"457.
8. Greenwood, Norman N.; Earnshaw, Alan , Chemistry of the Elements, 1997 (2nd ed.), Butterworth-Heinemann , ISBN 0080379419.

9. Rochelle M. Cornell, Udo Schwertmann, the Iron Oxides: Structure, Properties, Reactions, Occurrences and Uses, 2007, Wiley-VCH ISBN 3-527-60644-0

10. Ma, Ming; Zhang, Yu; Guo, Zhir4.ui; GU, Ning, Nanoscale Research Letters, 2013, 8 (1): 16. doi:10.1186/1556-276X-8-16.

11. Massart, R.; IEEE transactions on magnetics, 1981.,17, 2, 1247-1248

12. Sunggyu Lee (2006) Encyclopedia of Chemical Processing CRC Press ISBN 0-8247-5563-4

13. Valter, Ström; Richard T.; Olsson, K. V. Rao. 
J. Mater. Chem, 2010, 20, 4168-4175

14. Mei Fang, Valter Ström, Richard T. Olsson, Lyubov Belova, K. V. Rao, Rapid mixing: Appl. Phys. Lett.2011, 99, 222501

15. Monajjemi, M.; Baei, M.T.; Mollaamin, F. Russian Journal of Inorganic Chemistry. 2008, 53 (9), 1430-1437

16. Monajjemi, M.; Rajaeian, E.; Mollaamin, F.; Naderi, F.; Saki, S. Physics and Chemistry of Liquids. 2008, 46 (3), 299-306

17. Monajjemi, M.; Boggs, J.E. J. Phys. Chem. A, 2013, 117, 1670 "1684

18. Mollaamin, F.; Monajjemi, M, Journal of Computational and Theoretical Nanoscience. 2012, 9 (4) 597-601

19. Monajjemi, M.; Khaleghian, M, Journal of Cluster Science. 2011, 22 (4) , 673-692

20. Mollaamin, F.; Varmaghani, Z.; Monajjemi, M, Physics and Chemistry of Liquids. 2011, 49 318

21. Nafisi, S.; Monajemi, M.; Ebrahimi, S. Journal of Molecular Structure. 2004,705 (3) 35-39

22. Fazaeli, R.; Monajjemi, M.; Ataherian, F.; Zare, K. Journal of Molecular Structure: THEOCHEM.2002, 581 (1), 51-58

23. Monajjemi, M.; Razavian, M.H.; Mollaamin,F.; Naderi,F.; Honarparvar,B.; Russian Journal of Physical Chemistry A , 2008 , 82 (13), 22772285

24. Monajjemi, M.; Seyed Hosseini, M.; Mollaamin, F. Fullerenes, Nanotubes, and Carbon Nanostructures. 2013, 21, 381-393

25. Monajjemi, M.; Faham, R.; Mollaamin, F. Fullerenes, Nanotubes, and Carbon Nanostructures, 2012 20, 163-169

26. Mollaamin, F.; Najafi, F.; Khaleghian, M.; Khalili Hadad, B.; Monajjemi, M. Fullerenes, Nanotubes, and Carbon Nanostructures, 2011 19, 653-667

27. Mollaamin, F.; Baei, MT.; Monajjemi, M.; Zhiani, R.; Honarparvar, B.; Russian Journal of Physical Chemistry A, Focus on Chemistry, 2008, 82 (13), 2354-2361

28. Monajjemi, M. Chemical Physics. 2013, 425, 29-45

29. Monajjemi, M.; Heshmat, M.; Aghaei, H.; Ahmadi, R.; Zare, K. Bulletin of the Chemical Society of Ethiopia, 2007, 21 (1)

30. Monajjemi, M.; Honarparvar, B. H. ; Haeri, H. ; Heshmat ,M.; Russian Journal of Physical
Chemistry C. 2006, 80(1):S40-S44

31. Monajjemi, M.; Ketabi, S.; Amiri, A. Russian Journal of Physical Chemistry, 2006, 80 (1), S55-S62

32. Yahyaei, H.; Monajjemi, M.; Aghaie, H.; K. Zare, K. Journal of Computational and Theoretical Nanoscience. 2013, 10, 10, 2332-2341

33. Mollaamin, F.; Gharibe, S.; Monajjemi, M. Int. J. Phy. Sci, 2011, 6, 1496-1500

34. Monajjemi, M.; Ghiasi, R.; Seyed Sadjadi, M.A. Applied Organometallic Chemistry,2003, 17. 8, 635-640

35. Monajjemi, M.; Wayne Jr, Robert. Boggs, J.E. Chemical Physics. 2014, 433, 1-11

36. Monajjemi, M.; Sobhanmanesh, A.; Mollaamin, F. Fullerenes, Nanotubes, and Carbon Nanostructures, 2013, 21 47-63

37. Monajjemi, M.; Mollaamin, F. Journal of Computational and Theoretical Nanoscience, 2012, 9 (12) 2208-2214

38. Monajjemi, M.; Honarparvar, B.; Nasseri, S. M. .; Khaleghian M. Journal of Structural Chemistry. 2009, 50, 1, 67-77

39. Monajjemi, M.; Aghaie, H.; Naderi, F. Biochemistry (Moscow).2007, 72 (6), 652657

40. Ardalan, T.; Ardalan, P.; Monajjemi, M. Fullerenes, Nanotubes, and Carbon Nanostructures, 2014, 22: 687-708

41. Mollaamin, F.; Monajjemi, M.; Mehrzad, J. Fullerenes, Nanotubes, and Carbon Nanostructures. 2014, 22: 738-751

42. Monajjemi, M.; Najafpour, J.; Mollaamin, F. Fullerenes, Nanotubes, and Carbon Nanostructures. 2013, 21(3), 213-232

43. Monajjemi, M.; Karachi, N.; Mollaamin, F. Fullerenes, Nanotubes, and Carbon Nanostructures, 2014, 22: 643-662

44. Yahyaei, H.; Monajjemi, M. Fullerenes, Nanotubes, and Carbon Nanostructures.2014, 22(4), 346-361

45. Monajjemi, M. Falahati, M.; Mollaamin, F.; Ionics, 2013, 19, 155-164

46. Monajjemi, M.; Mollaamin, F. Journal of Cluster Science, 2012, 23(2), 259-272

47. Tahan, A.; Monajjemi, M. Acta Biotheor, 2011, 59, 291-312

48. Lee, V.S.; Nimmanpipug, P.; Mollaamin, F.; Kungwan, N.; Thanasanvorakun, S..; Monajjemi, M. Russian Journal of Physical 
Chemistry A, 2009, 83, 13, 2288-2296

49. Monajjemi, M.; Heshmat, M.; Haeri, HH, Biochemistry (Moscow), 2006, 71 (1), S113S122

50. Monajjemi, M.; Yamola, H.; Mollaamin, F. Fullerenes, Nanotubes, and Carbon Nanostructures, 2014, 22, 595-603

51. Mollaamin, F.; Layali, I.; Ilkhani A. R.; Monajjemi, M. African Journal of Microbiology Research .2010, 4(24) 2795-2803

52. Mollaamin, F.; Shahani poor, p K. .; Nejadsattari, T. ; Monajjemi, M. African Journal of Microbiology Research. 2010, 4(20) 2098-2108

53. Monajjemi, M.; Ahmadianarog, M. Journal of Computational and Theoretical Nanoscience. 2014, 11(6), 1465-1471

54. Monajjemi, M.; Jafari Azan, M.; Mollaamin, F. Fullerenes, Nanotubes, and Carbon Nanostructures.2013, 21(6), 503-515

55. Mollaamin, F.; Monajjemi, M. Physics and Chemistry of Liquids .2012, 50, 5, 2012, 596-604

56. Monajjemi, M.; Khosravi, M.; Honarparvar, B.; Mollaamin, F.; International Journal of Quantum Chemistry, 2011, 111, 27712777

57. Khaleghian, M.; Zahmatkesh, M.; Mollaamin, F.; Monajjemi, M. Fullerenes, Nanotubes, and Carbon Nanostructures, 2011, 19(4): 251-261

58. Monajjemi, M.; Baheri, H.; Mollaamin, F. Journal of Structural Chemistry.2011 52(1), 54-59

59. Mahdavian, L.; Monajjemi, M.; Mangkorntong, N. Fullerenes, Nanotubes and Carbon Nanostructures, 2009, 17 (5), 484-495

60. Monajjemi, M., Mahdavian, L., Mollaamin, F. Bull. Chem. Soc. Ethiop. 2008, 22(2), 277286

61. Monajjemi, M.; Afsharnezhad, S, Jaafari, M.R..; Mirdamadi, S..; Mollaamin, F..; Monajemi, $\mathrm{H}$. Chemistry .2008, 17 (1), 55-69

62. Monajjemi, M.; Mollaamin, F.; Gholami, M. R.; Yoozbashizadeh, H.; Sadrnezhaad, S.K.; Passdar, H.; Main Group Metal Chemistry, 2003, 26, 6, 349-361

63. Monajjemi, M.; Azad ,MT.; Haeri, HH.; Zare, K.; Hamedani, Sh.; JOURNAL OF CHEMICAL RESEARCH-S.2003, (8): 454-456
64. Monajjemi, M.; Najafpour, J. Fullerenes, Nanotubes, and Carbon Nanostructures, 2014, 22(6): 575-594

65. Monajjemi, M.; Noei, M.; Mollaamin, F. Nucleosides, Nucleotides and Nucleic Acids. 2010 29(9):676-683

66. Ghiasi, R.; Monajjemi, M. Journal of Sulfur Chemistry . 28, 5, 505-511

67. Monajjemi, M.; Ghiasi, R.; Abedi, A. Russian Journal of Inorganic Chemistry.2005, 50(3), 382-388

68. Monajjemi, M. .; Naderi, F.; Mollaamin, F.; Khaleghian, M. J. Mex. Chem. Soc. 2012, 56(2), 207-211

69. Monajjemi, M.; Farahani, N.; Mollaamin, F. Physics and Chemistry of Liquids, 2012, 50(2) 161-172

70. Monajjemi, M.; Seyed Hosseini, M. Journal of Computational and Theoretical Nanoscience .2013, 10 (10), 2473-2477

71. Monajjemi , M.; Honaparvar, B.; Khalili Hadad ,B.; Ilkhani ,AR.; Mollaamin, F. African Journal of Pharmacy and Pharmacology .2010, 4(8), $521-529$

72. Monajjemi, M. Theor Chem Acc, 2015, 134:77 DOI 10.1007/s00214-015-1668-9

73. Monajjemi, M. Journal of Molecular Modeling , 2014, 20, 2507

74. Monajjemi , M.; Honarparvar, B.; Monajemi, H.;. Journal of the Mexican Chemical Society, 2006, 50 (4), 143-148

75. Monajjemi, M.; Khaleghian, M.; Mollaamin, F. Molecular Simulation. 2010, 36, 11, 865-

76. Ilkhani, Ali R.; Monajjemi, M. Computational and Theoretical Chemistry.2015 1074, 19-25

77. Monajjemi, M. Biophysical Chemistry. 2015 207,114-127

78. Monajjemi, M., Moniri, E., Panahi, H.A , Journal of Chemical and Engineering Data.2001, 1249-1254.

79. Mollaamin, F.; Najafpour, J.; Ghadami, S.; Ilkhani, A. R.; Akrami, M. S.; Monajjemi, M. Journal of Computational and Theoretical Nanoscience. 11 (5), 1290-1298

80. Monajjemi, M.; Ghiasi, R.; Ketabi, S.; Passdar, H.; Mollaamin, F. Journal of Chemical Research . 2004, 1, 11.

81. Monajjemi, M.; Heshmat, M.; Haeri, H.H. Biochemistry (Moscow).2006, 71, 113-122 
82. Monajjemi, M.; Heshmat, M.; Aghaei, H.;Ahmadi, R.; Zare, K. Bulletin of the Chemical Society of Ethiopia. 2007, 21, 111-116

83. Monajjemi, M., Kharghanian, L., Khaleghian, M., Chegini, H. Fullerenes Nanotubes and Carbon Nanostructures.2014, 22, 8, 0.1080/1536383X.2012.717563

84. Sarasia, E.M.; Afsharnezhad, S.; Honarparvar, B.; Mollaamin, F.; Monajjemi, M.Physics and Chemistry of Liquids. 2011, 49 (5), 561-571

85. Amiri, A.; Babaeie, F.; Monajjemi, M. Physics and Chemistry of Liquids. 2008, 46, 4, 379389

86. Monajjemi, M.; Heshmat, M.; Haeri, H.H.; Kaveh, F. Russian Journal of Physical Chemistry A, 2006, 80, 7, 1061-1068

87. Monajjemi, M.; Moniri, E.; Azizi, Z.; Ahmad Panahi, H. Russian Journal of Inorganic Chemistry. 2005, 50, 1, 40-44

88. Jalilian,H.; Monajjemi, M. Japanese Journal of Applied Physics. 2015, 54, 8, 08510

89. Mollaamin, F.; Monajjemi, M. Journal of Computational and Theoretical Nanoscience.2015, 12, 6, 1030-1039

90. Felegari, Z.; Monajjemi, M. Journal of Theoretical and Computational Chemistry. 2015, 14, 3, 1550021

91. Shojaee, S., Monajjemi, M. Journal of Computational and Theoretical Nanoscience.2015, 12, 3, 449-458

92. Esmkhani, R.; Monajjemi, M. Journal of Computational and Theoretical Nanoscience.2015. 12, 4, 652-659

93. Monajjemi, M., Seyedhosseini, M., Mousavi, M., Jamali, Z. Journal of Computational and Theoretical Nanoscience. 2015 , 23 (3), 239244

94. Ghiasi, R.; Monajjemi, M.; Mokarram, E.E.; Makkipour, P. Journal of Structural Chemistry.2008, 4 , 4, 600-605

95. Mahdavian, L.; Monajjemi, M. Microelectronics Journal. 2010, 41(2-3), 142-149

96. Monajjemi, M.; Baie, M.T.; Mollaamin, F. Russian Chemical Bulletin.2010, 59, 5, 886889

97. Bakhshi, K.; Mollaamin, F.; Monajjemi, M. Journal of Computational and Theoretical Nanoscience. 2011, 8, 4, 763-768

98. Darouie, M.; Afshar, S.; Zare, K., Monajjemi,
M. journal of Experimental Nanoscience.2013, 8, 4, 451-461

99. Amiri, A.; Monajjemi, M.; Zare, K.; Ketabi, S. Physics and Chemistry of Liquids. 2006, 44, 4, 449-456.

100. Zonouzi, R.;Khajeh, K.; Monajjemi, M.; Ghaemi, N. Journal of Microbiology and Biotechnology. 2013, 23, 1 ,7-14

101. Ali R. Ilkhani, Majid Monajjemi, Computational and Theoretical Chemistry 1074 (2015) 19-25

102. Tahan, A.; Mollaamin, F.; Monajjemi, M. Russian Journal of Physical Chemistry A, 2009, 83 (4), 587-597

103. Khalili Hadad, B.; Mollaamin, F.; Monajjemi, M, Russian Chemical Bulletin,2011, 60(2):233236

104. Mollaamin, F.; Monajjemi, M.; Salemi, S.; Baei, M.T. Fullerenes Nanotubes and Carbon Nanostructures, 2011, 19, 3, 182-196

105. Mollaamin, F.; Shahani Pour.; K., Shahani Pour, K.; ilkhani, A.R.; Sheckari, Z., Monajjemi, M Russian Chemical Bulletin , 2012 , 61(12), 2193-2198

106. Shoaei, S.M.; Aghaei, H.; Monajjemi, M.; Aghaei, M. Phosphorus, Sulfur and Silicon and the Related Elements. 2014, 189, 5; 652660

107. Mehrzad, J., Monajjemi, M., Hashemi, M , Biochemistry (Moscow).2014 , 79 (1), 31-36

108. Moghaddam, N.A., Zadeh, M.S., Monajjemi, M. Journal of Computational and Theoretical Nanoscience , 2015 , 12,. 3, doi:10.1166/ jctn.2015.3736

109. Joohari, S.; Monajjemi, M , Songklanakarin Journal of Science and Technology , 2015 , 37(3):327

110. Rajaian, E., Monajjemi, M., Gholami, M.R, Journal of Chemical Research - Part S,2002, $6,1,279-281$

111. Ghassemzadeh, L., Monajjemi, M., Zare, K, Journal of Chemical Research - Part S, 2003, 4, 195-199

112. Monajjemi, M.; Lee, V.S.; Khaleghian, M.; B. Honarparvar, B.; F. Mollaamin, F. J. Phys. Chem C. 2010, 114, 15315

113. Monajjemi, M. Struct Chem. 2012, 23,551580

114. Monajjemi, M.; Chegini, H.; Mollaamin, F.; Farahani, P. Fullerenes, Nanotubes, and 
Carbon Nanostructures. 2011, 19, 469-482

115. Monajjemi, M .; Afsharnezhad ,S.; Jaafari , M.R.; Abdolahi ,T.; Nikosade ,A.; Monajemi ,H.; Russian Journal of physical chemistry A, 2007, 2,1956-1963

116. Mehdizadeh Barforushi,M.; Safari,S.; Monajjemi,M. J. Comput. Theor. Nanosci.2015, 12, 3058-3065.

117. Mollaamin,F.; Ilkhani,A.; Sakhaei,N.; Bonsakhteh,B.; Faridchehr,A.; Tohidi,S.; Monajjemi, M. J. Comput. Theor. Nanosci. 2015, 12, 3148-3154

118. Rahmati,H.; Monajjemi,M. J. Comput. Theor. Nanosci. 2015, 12, 3473-3481.

119. Roghieh Tarlani Bashiz,R.; Monajjemi,M. J. Comput. Theor. Nanosci.2015, 12, 38083816

120. Mehrabi Nejad,A.; Monajjemi,M. J. Comput. Theor. Nanosci. 2015, 12, 3902-3910.

121. Monajjemi,M.; Bagheri,S.; Moosavi,M.S.; Moradiyeh,N.; Zakeri,M.;Attarikhasraghi,N.; Saghayimarouf, N.; Niyatzadeh,G.; Shekarkhand,M.; Khalilimofrad,M.S.; Ahmadin,H.; Ahadi,M.; Molecules 2015, 20, 21636-21657; doi:10.3390/ molecules201219769

122. Shabanzadeh,E.; Monajjemi,M.; J. Comput. Theor. Nanosci.2015, 12, 4076-4086

123. Elsagh,A.; Jalilian, H.; Kianpour, E.; Ghazi, Mokri, H.S.; Rajabzadeh,M.; Moosavi,M.S.; Ghaemi Amiri,F.; Majid Monajjemi ,M.; J. Comput. Theor. Nanosci. 2015, 12, 42114218.

124. Faridchehr, A.; Rustaiyan,A.; Monajjemi,M.; J. Comput. Theor. Nanosci.2015, 12, 43014314.

125. Tohidi, S.; Monajjemi, M.; Rustaiyan, A.; J. Comput. Theor. Nanosci. 2015, 12, 43454351

126. Ali Akbari Zadeh,M.; Lari,H.; Kharghanian,L.; Balali,E.; Khadivi,R.;Yahyaei,H.; Mollaamin,F.; Monajjemi, M.; J. Comput. Theor. Nanosci.2015, 12, 4358-4367.

127. Dezfooli,S.; Lari,H.; Balali,E.; Khadivi,R.; Farzi,F.; Moradiyeh,N.; Monajjemi,M.; J. Comput. Theor. Nanosci.2015, 12, 4478-4488
128. Jalilian,H.; Sayadian,M.; Elsagh,A.; Farzi,F.; Moradiyeh,N.; Samiei Soofi,N.; Khosravi,S.; Mohammadian,N.T.; Monajjemi,M. J. Comput. Theor. Nanosci. 2015, 12, 4785-4793.

129. Farzi,F.; Bagheri,S.; Rajabzadeh,M.; Sayadian,M.; Jalilian,H.; Moradiyeh,N.; Monajjemi, M.; J. Comput. Theor. Nanosci.2015, 12, 4862-4872.

130. Monajjemi,M.; Mohammadian, N.T, J. Comput. Theor. Nanosci. 2015, 12, 4895-4914

131 Monajjemi, M., Chahkandi, B. Journal of Molecular Structure: THEOCHEM, 2005, 714 (1), 28, 43-60.

131. Naghsh,F, Orient. J. Chem., 2015, 31(1)., 465-478

132. Chitsazan, A, Orient. J. Chem., 2015, 31(1)., 393-408

133. R.F.W. Bader, atoms in Molecule: A quantum Theory (Oxford Univ. press, Oxford, 1990).

134. Savin, A., Jepsen,O., Flad, J., Andersen, OK, Preuss, H. \& von Schering, HG. Angew. Chem. Int. Ed. Engl. 1992, 31, 187-188

135. Tsirelson and Stash, Chem. Phys. Lett. 2002 , 351, 142

136. Schmider and Becke, J. Mol. Struct. (THEOCHEM), 2000, 527, 51.

137. Heiko Jacobsen, Canadian Journal of Chemistry, 2009, 87(7): 965-973, 10.1139/ v09-060

138. Sadegh,H.; Shahryari-ghoshekandi,R.; Agrawal, S.; Tyagi,I.; Asif,M, Gupta,V.K, Journal of Molecular Liquids, 2015, 206, 151158 .

139. Gupta,V.K.; Tyagi,I.; Agrawal,S.; Sadegh,H.; Shahryari-ghoshekandi, R.; Yari,M.; Yousefinejat,O.; Journal of Molecular Liquids, 2015, 206, 126-136

140. Sadegh,H.; Shahryari-ghoshekandi,R.; Tyagi,I.; Agrawal,S.; Gupta,V.K, Journal of Molecular Liquids,2015, 207, 21-27.

141. Sadegh,H.:Zare,K.; Maazinejad,B.; Shahryarighoshekandi,R.; Tyagi,I.; Agrawal,S.; Gupta,V.K, Journal of Molecular Liquids, 2016,215, 221-228.

142. Zare,K.; Sadegh,H.; Shahryarighoshekandi,R.; Asif,M.; Tyagi,I.; Shilpi Agrawal, Gupta,V.K Journal of Molecular Liquids, 2016, 213, 345-350 